\title{
Analogies between the topologial insulator phase of 2D Dirac materials and the superradiant phase of atom-field systems
}

\author{
Manuel Calixto ${ }^{1}$, Elvira Romera ${ }^{1}$, and Octavio Castaños ${ }^{2}$ \\ ${ }^{1}$ Universidad de Granada \\ ${ }^{2}$ Universidad Nacional Autonoma de Mexico
}

May 5, 2020

\begin{abstract}
A semiclassical phase-space perspective of band- and topological-insulator regimes of 2D Dirac materials, and normal- and superradiant-phases of atom-field interacting models is given in terms of delocalization, entropies, and quantum correlation measures. From this point of view, the low-energy limit of tight-binding models describing the electronic band structure of topological 2D Dirac materials like phosphorene and silicene with tunable band gaps, share similarities with Rabi-Dicke and Jaynes-Cummings atom-field interaction models, respectively. In particular, the edge state of 2D Dirac materials in the topological insulator phase exhibits a Schrödinger cat structure similar to the ground state of two-level atoms in a cavity interacting with a one-mode radiation field in the superradiant phase. Delocalization seems to be a common feature of topological insulator and superradiant phases.
\end{abstract}

\section{Hosted file}

PhosphoreneHusimi_IJQC.pdf available at https://authorea.com/users/303463/articles/433586analogies-between-the-topologial-insulator-phase-of-2d-dirac-materials-and-thesuperradiant-phase-of-atom-field-systems

\section{Hosted file}

PhosphoreneHusimi_IJQC.tex available at https://authorea.com/users/303463/articles/433586analogies-between-the-topologial-insulator-phase-of-2d-dirac-materials-and-thesuperradiant-phase-of-atom-field-systems 

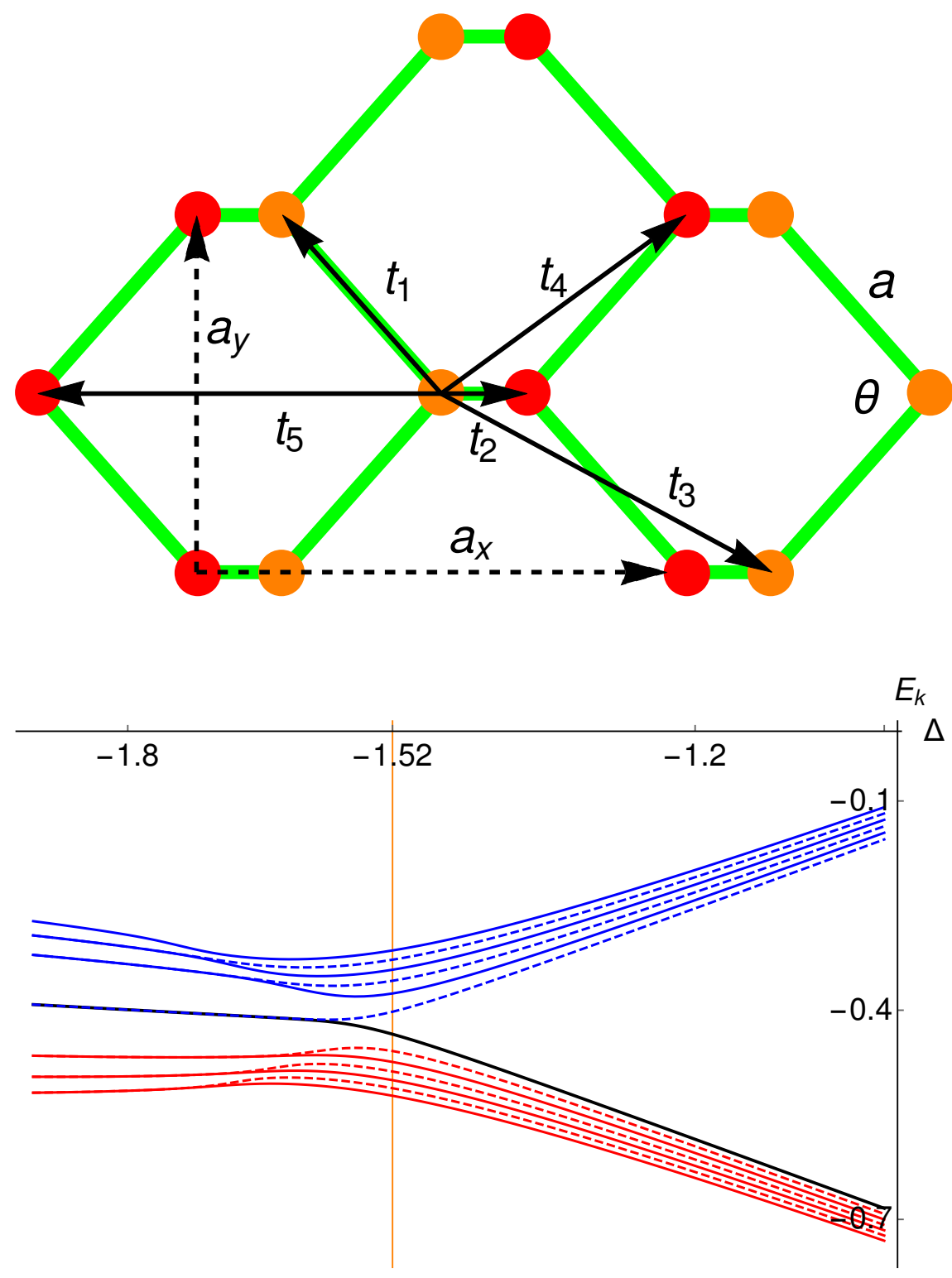

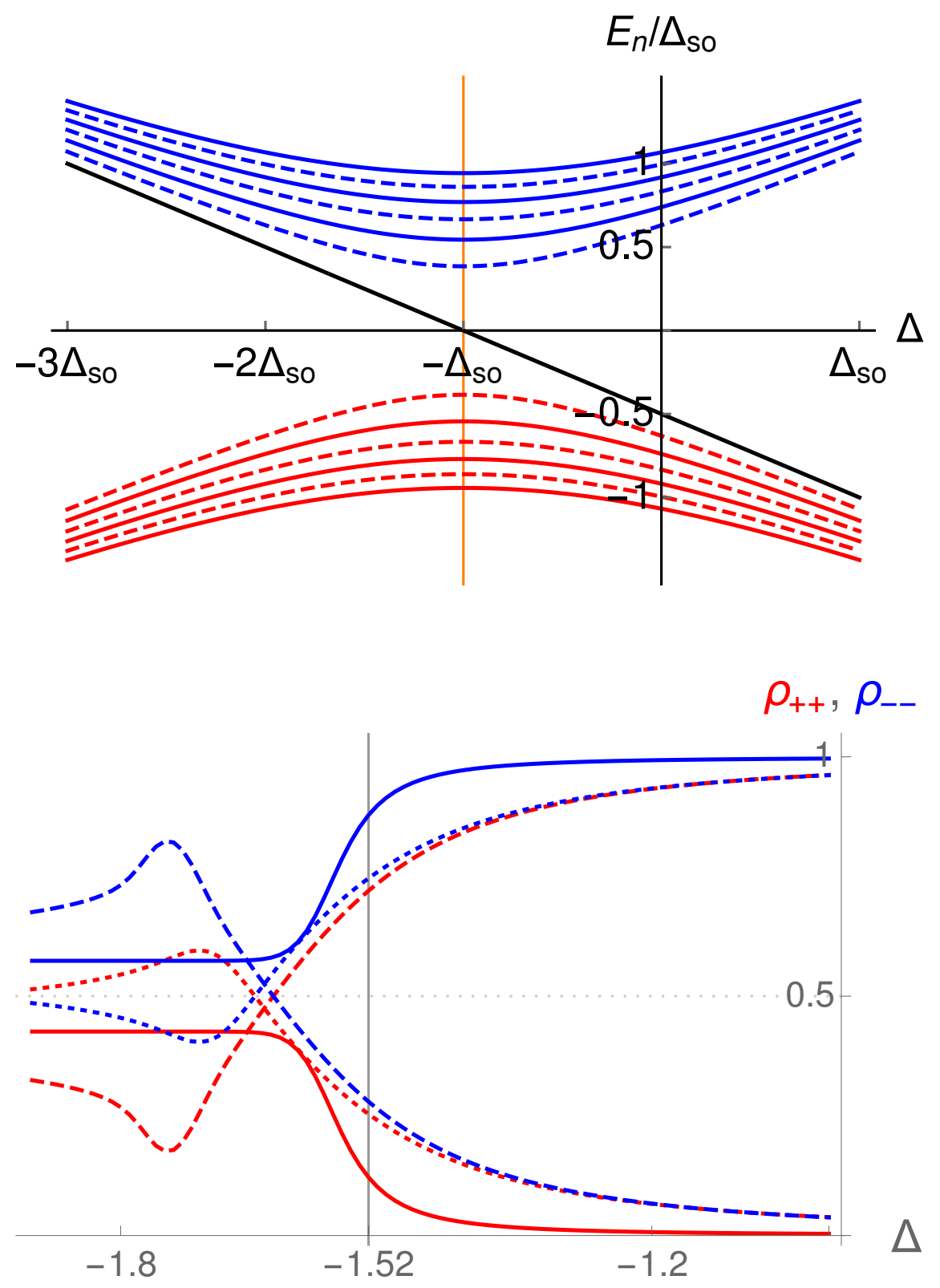

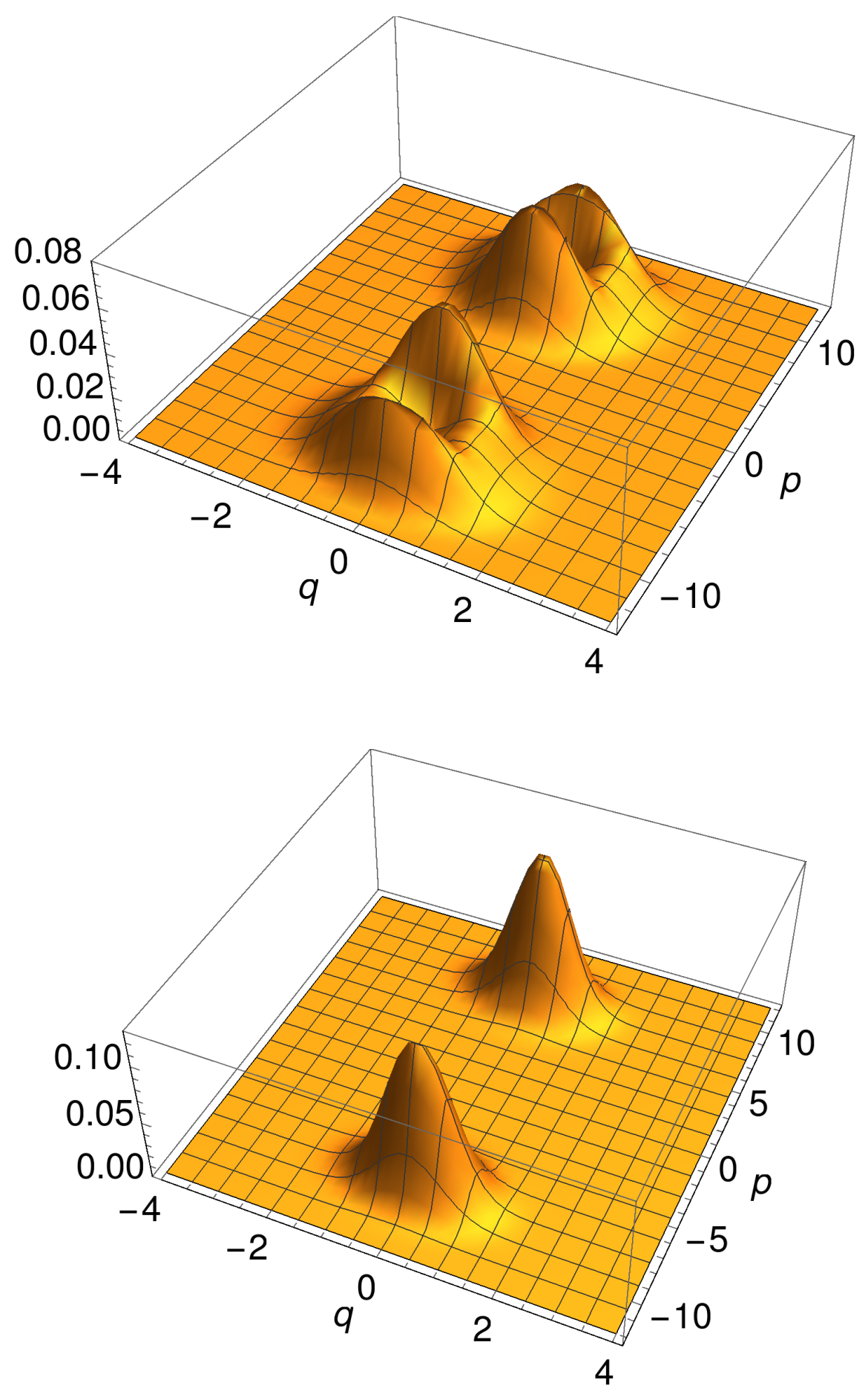

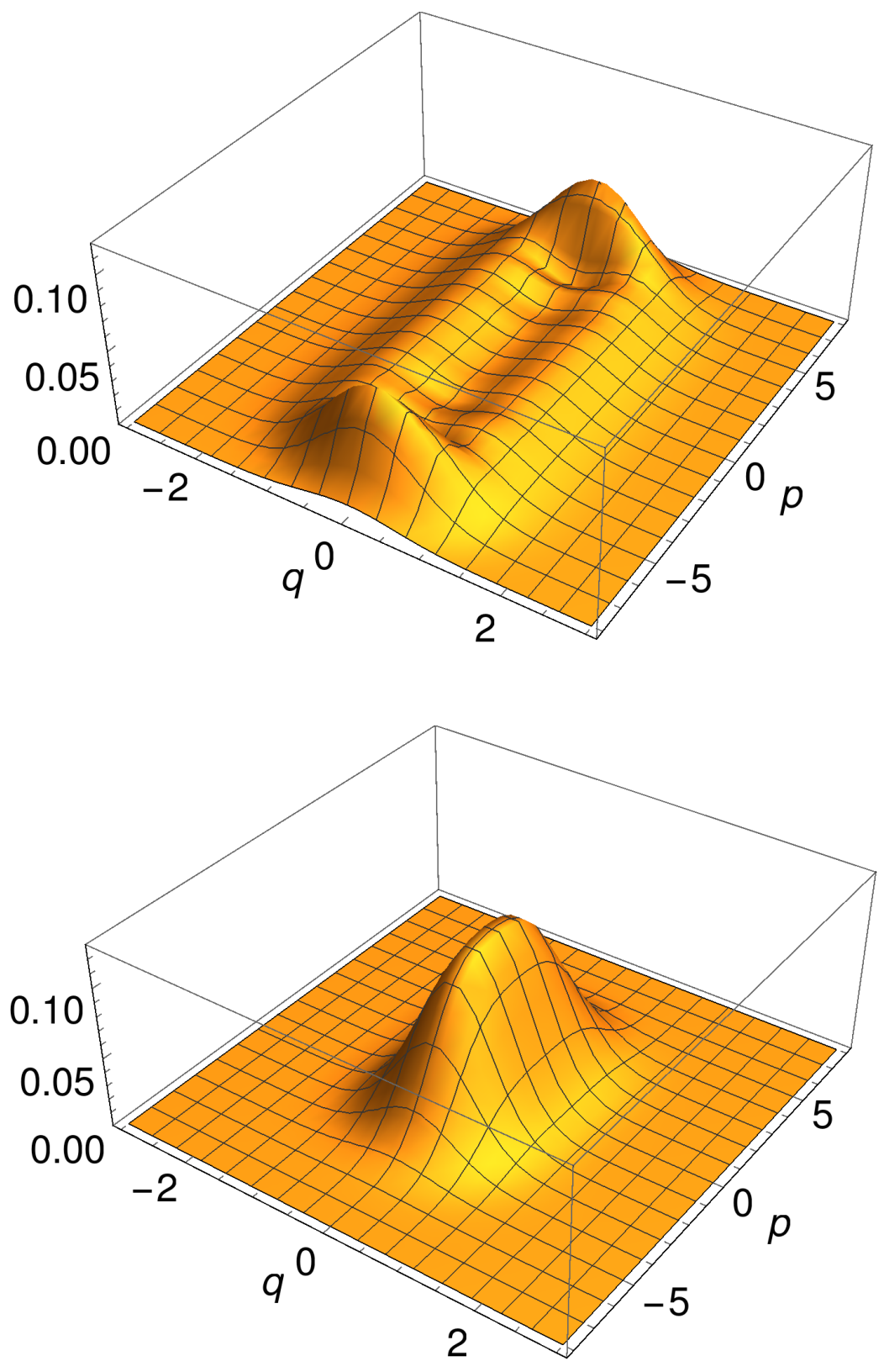

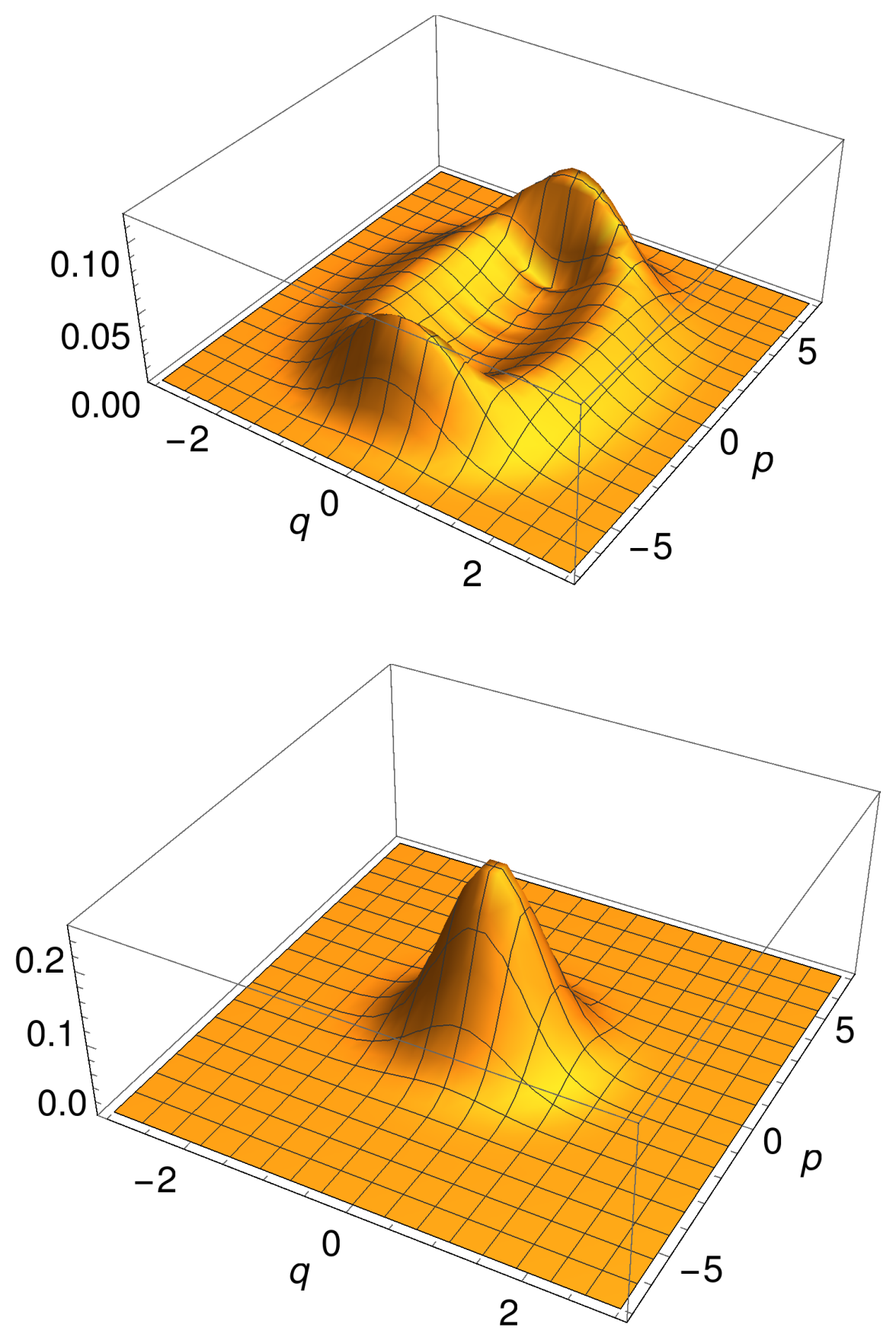

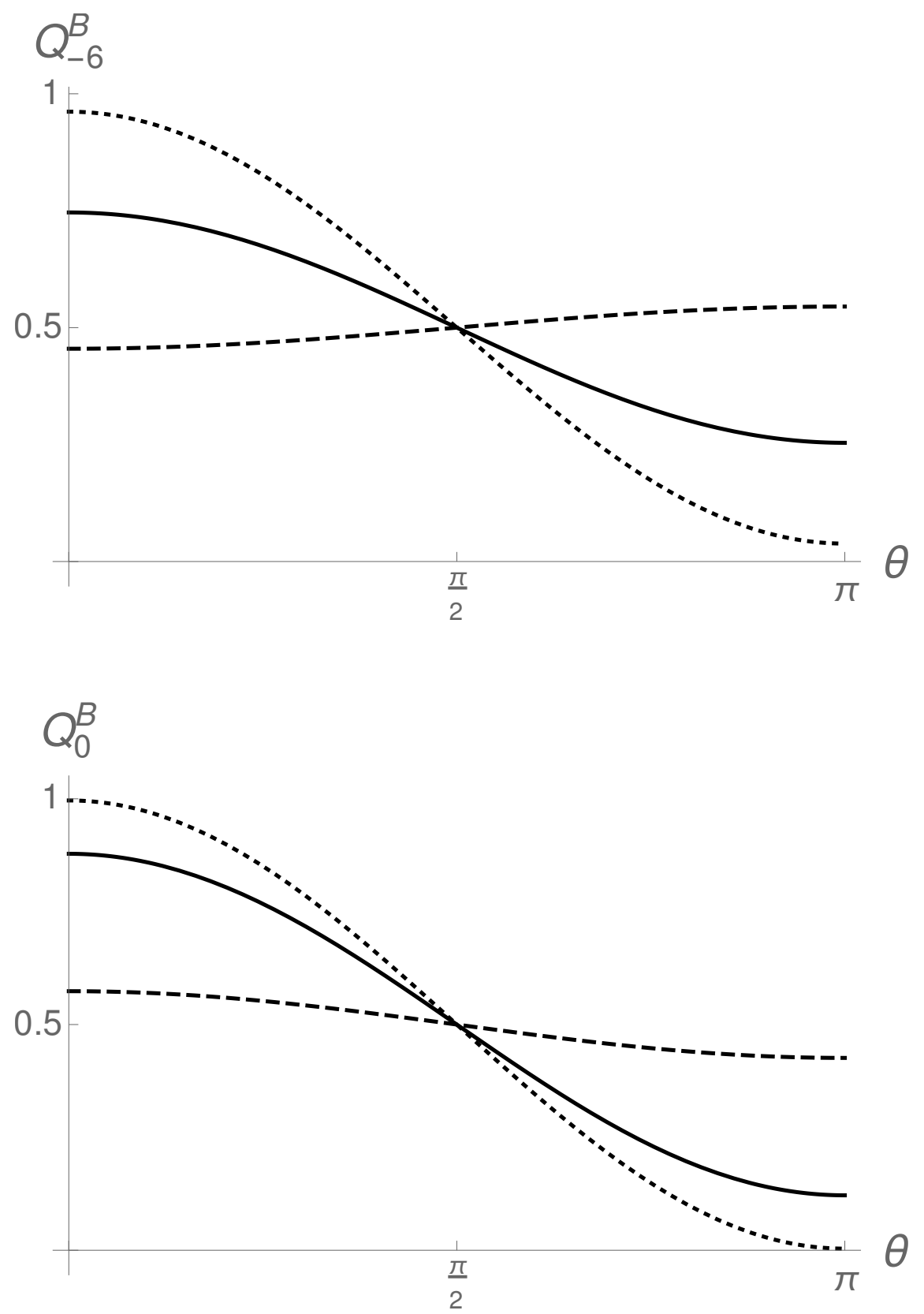

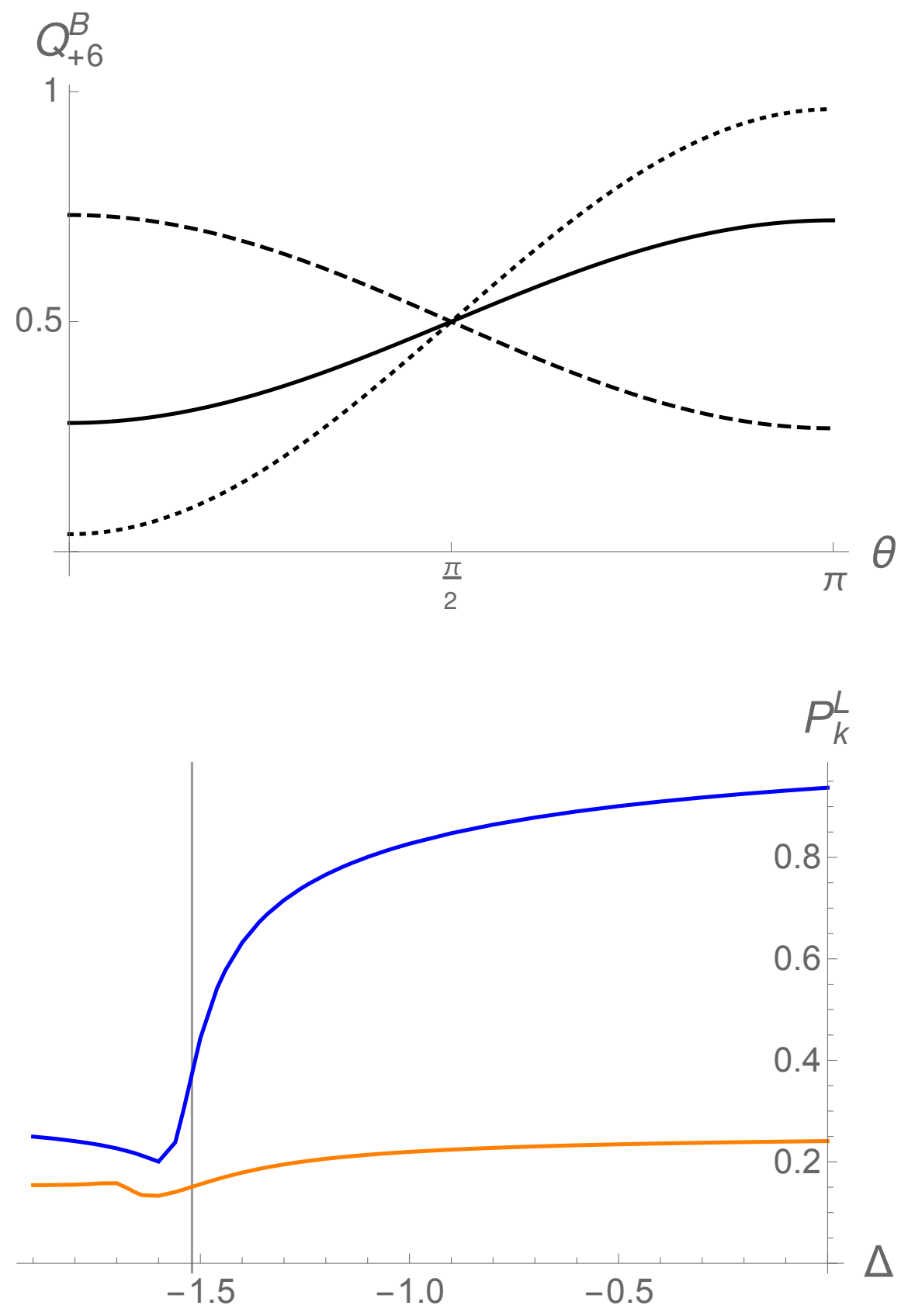


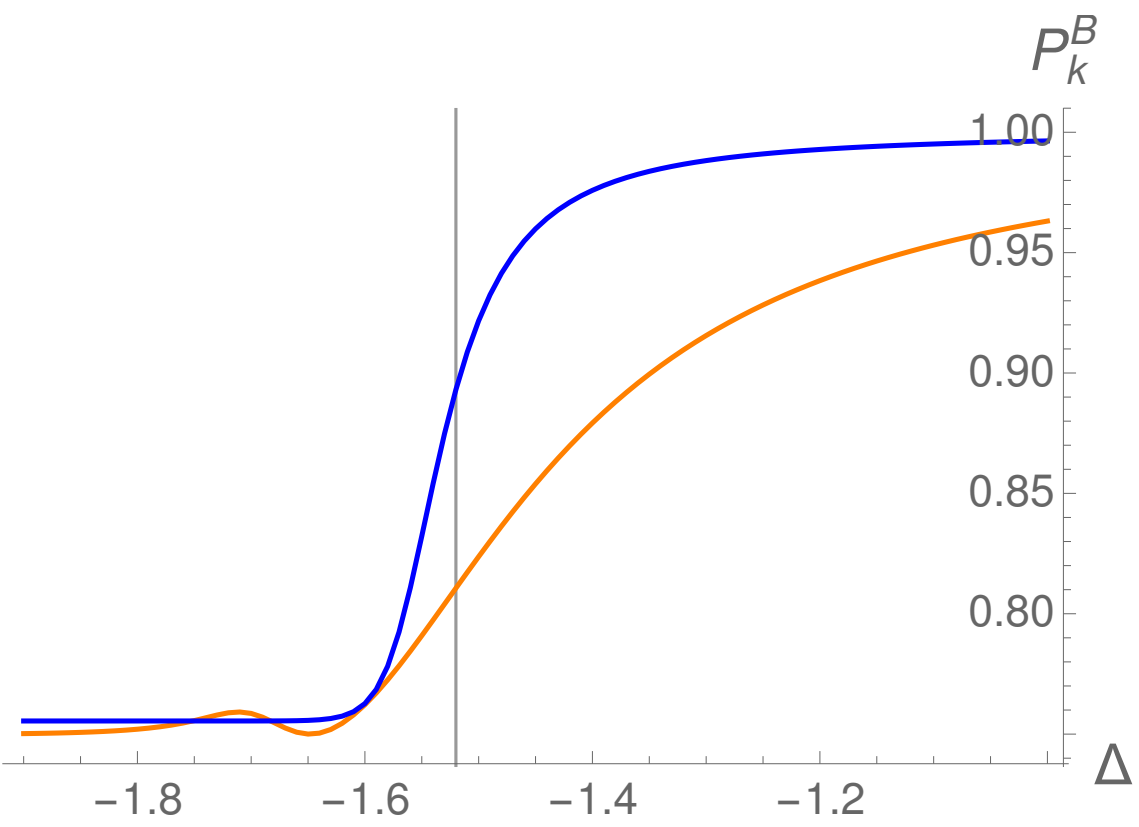

